\title{
Evaluation of Tumor Markers and Their Impact on Prognosis in Gallbladder, Bile Duct and Cholangiocellular Carcinomas - A Pilot Study
}

\author{
VACLAV LISKA ${ }^{1,2}$, VLADISLAV TRESKA ${ }^{1}$, TOMAS SKALICKY ${ }^{1}$, JAKUB FICHTL ${ }^{1}$, \\ JAN BRUHA ${ }^{1,2}$, ONDREJ VYCITAL ${ }^{1,2}$, ONDREJ TOPOLCAN ${ }^{3}$, RICHARD PALEK ${ }^{1,2}$, \\ JACHYM ROSENDORF ${ }^{1,2}$, JIRI POLIVKA ${ }^{2,4}$ and LUBOS HOLUBEC ${ }^{2}$ \\ ${ }^{1}$ Department of Surgery, Faculty of Medicine in Plzen, Charles University, Plzen, Czech Republic; \\ ${ }^{2}$ Biomedical Center, Faculty of Medicine in Plzen, Charles University, Plzen, Czech Republic; \\ ${ }^{3}$ Central Immunoanalytical Laboratory, Faculty Hospital Plzen, Plzen, Czech Republic; \\ ${ }^{4}$ Department of Histology and Embryology, Faculty of Medicine in Plzen, Charles University, Plzen, Czech Republic
}

\begin{abstract}
Background/Aim: The behavior of tumor markers in biliary tract malignancies is not well-known and has been scarcely studied. Such markers could play important roles in diagnostic and prognostic schemes as well as in decision-making about the best treatment strategies. This study analyzed the preoperative serum levels of conventional tumor markers (AFP, CEA, CA 19-9, $C A$ 72-4), proliferative marker thymidine kinase (TK) and cytokeratins (TPA, TPS and CYFRA 21.1) in patients with gallbladder carcinoma, bile duct carcinoma (Klatskin) and cholangiocellular carcinoma, in relation to the patient prognosis. The study aimed in finding the role of tumor markers in not properly investigated diseases, where their importance is often marginalized. Materials and Methods: The study included 43 patients, who underwent either radical surgical procedure $(n=21)$ or explorative laparotomy without any surgical treatment $(n=22)$ for gallbladder carcinoma, bile duct carcinoma (Klatskin tumor) and cholangiocellular carcinoma $(24,8$ and 11 patients, respectively) between 2003 and 2010 at our Department. The association of serum tumor markers and patients' prognosis were assessed for the entire cohort and for each cancer type and also with regard to treatment (radical surgery versus explorative laparotomy). Overall survival (OS) and disease-free interval (DFI) were estimated by the Kaplan-Meier method and statistically
\end{abstract}

Correspondence to: Vaclav Liska, MD, Ph.D., Department of Surgery, Faculty of Medicine in Plzen, Charles University, alej Svobody 80, 30460 Plzen, Czech Republic. E-mail: vena.liska@gmail.com

Key Words: Gall bladder carcinoma, bile duct carcinoma, cholangiocellular carcinoma, liver surgery, tumor markers, prognosis. evaluated using the LogRank test. DFI was computed only in the subgroup of patients treated by radical surgery. Results: The statistical analysis of tumor markers revealed $T K$ as a poor prognostic factor for shorter DFI (HR=3.5, $95 \% C I=0.6-21.3, \quad p<0.05)$ and also $O S \quad(H R=4.6$, $95 \% C I=1.0-4.7, p<0.05)$ in patients with gallbladder carcinoma treated with radical surgery. TPS was demonstrated as a poor prognostic factor for OS in patients with gallbladder carcinoma ( $H R=12.7,95 \% C I=1.4-117.7$, $p<0.05)$. CEA was proven to be a factor of poor prognosis with shorter OS in patients after explorative laparotomy for all cumulated studied diagnoses (HR=9.8, 95\%CI=1.0592.7, p<0.05). Conclusion: The results of this study suggested the importance of tumor markers for assessment of prognosis (OS or DFI) in patients with gallbladder carcinoma, bile duct carcinoma, and cholangiocellular carcinoma.

The surgical treatment of liver and biliary tract malignancies was powered in last two decades by a number of new technical possibilities and skills. Also the perioperative intensive care for patients after extended liver and biliary tract resections and large biliary tract reconstructions was significantly improved. The number of patients that undergo radical surgery for gallbladder, extrahepatic bile duct (Klatskin) and cholangiocellular carcinomas is substantially increasing as well. Despite these improvements, early recurrences are detected in a majority of patients after radical treatment. The prognosis of patients with early recurrence is extremely unfavorable. Even after curative resection of the primary tumor, the long-term prognosis remains poor. The five-year survival for gallbladder and biliary tract carcinomas are only $5-12 \%$ with the best results for cholangiocarcinoma (1-4). 
Due to the rare incidence of the gallbladder, extrahepatic bile duct and cholangiocellular carcinomas $(1.2 / 100,000$, $1.5 / 100,000$ and $1.5 / 100,000$ respectively) there is a relative lack of large studies evaluating the prognostic and predictive biomarkers for these diseases (3-5). The predictors of longterm survival (more than 24 months) after radical treatment of gallbladder carcinoma were identified as localized disease without lymph nodes metastases, adjuvant oncological treatment, R0 resection, low grading of the tumor and absence of vascular invasion $(6,7)$.

Despite different clinical behavior of gallbladder, extrahepatic bile duct and cholangiocarcinoma, all these malignancies histologically originate from epithelia of biliary tract deriving from epithelia of primary duodenum during the embryogenetic process $(8,9)$. Thus the immunohistochemical patterns of these cancers are often very similar or even the same (10). Research of tumor markers in relation to the gallbladder, extrahepatic bile duct and cholangiocellular carcinomas is still insufficient. This situation is caused mainly by the rare incidence of these tumors in surgical departments as well as a long time needed for the collection of appropriate patient cohorts.

This study evaluated the impact of serum levels of conventional tumor markers (AFP, CEA, CA19-9, CA72-4), proliferative marker thymidine kinase (TK) and cytokeratins (TPA, TPS and CYFRA 21-1) on prognosis (overall survival - OS and disease-free interval - DFI) in our cohort of patients with gallbladder, extrahepatic bile duct and cholangiocellular carcinomas. The sub-analyses were performed respecting the distinct tumor types and the extent of therapeutic intervention (radical surgery $v s$. explorative laparotomy).

\section{Patients and Methods}

Patients. The study cohort included 43 patients with biliary tract malignancies who were treated either by radical surgical intervention $(n=21)$ or underwent explorative laparotomy $(n=22)$ between 2003 and 2010 at the Department of Surgery, University Hospital and Faculty of Medicine in Pilsen, Charles University. Among 24 patients with gallbladder carcinoma, 11 subjects were treated by radical surgery, whereas 13 patients experienced explorative laparotomy. Among 8 patients with extrahepatic bile duct carcinoma (Klatskin tumor), 5 patients underwent radical surgery and 3 patients experienced explorative laparotomy. Among 11 patients with cholangiocellular carcinoma, 5 were treated by radical surgery and 6 underwent explorative laparotomy for inoperable carcinoma (Table I). There were 18 men and 25 women in the entire cohort. The median follow-up of patients was 12 months (range 1-26 months). The average patient age was 55 years (range=29-79 years). The study was performed retrospectively.

Serum samples and immunological methods. Blood samples for assessments of tumor markers were collected at standard conditions during the morning hours from patient's cubital vein before surgery. The serum was acquired through centrifugation and stored until
Table I. Overview of cancer types and surgical treatment approaches in the entire study cohort.

\begin{tabular}{lccc}
\hline Tumor type & $\mathrm{N}$ & $\begin{array}{c}\text { Radical } \\
\text { treatment }\end{array}$ & $\begin{array}{c}\text { Explorative } \\
\text { laparotomy }\end{array}$ \\
\hline Gallbladder carcinoma & 24 & 11 & 13 \\
$\begin{array}{l}\text { Bile duct carcinoma } \\
\text { (Klatskin) }\end{array}$ & 8 & 5 & 3 \\
$\begin{array}{l}\text { Cholangiocarcinoma } \\
\text { (intrahepatic) }\end{array}$ & 11 & 5 & 6 \\
\hline
\end{tabular}

Table II. Overview of immunoanalytical assays used for each tumor marker.

\begin{tabular}{lccc}
\hline Tumor marker & Units & Assay & Manufacturer \\
\hline AFP & ng/ml & IRMA & Beckman Coulter \\
CEA & ng/ml & LIA & Beckman Coulter \\
CA 19-9 & IU/ml & LIA & Beckman Coulter \\
CA 72-4 & IU/ml & IRMA & Cis Bio International \\
Cyfra 21.1 & ng/ml & IRMA & Beckman Coulter \\
TPA & IU/1 & IRMA & Beki \\
TPS & IU/1 & IRMA & IDL \\
TK & IU/1 & REA & Immunotech \\
\hline
\end{tabular}

laboratory analysis at a temperature of $-20^{\circ} \mathrm{C}$. The serum levels of conventional tumor markers (AFP, CEA, CA 19-9, CA 72-4) as well as proliferative and cytokeratin tumor markers (TK, CYFRA 21-1, TPA and TPS) were determined using standardized commercially available immunoanalytical assays at the immunoanalytical laboratory of the University Hospital Pilsen. Assays were measured in different formats, including immunoradiometric (IRMA) assay, radioenzymatic (REA) assay and Line immunoassay (LIA). The concentrations of particular tumor markers were measured by following immunoanalytical methods from different producers: AFP - IRMA (Beckman Coulter, Miami, FL, US); CEA- LIA (Beckman Coulter); CA19-9 - LIA (Beckman Coulter); CA72-4 - IRMA, Cis Bio International, Versailles, France); CYFRA 21-1 - IRMA (Beckman Coulter); TPA - IRMA (BEKI Diagnostics AB, Solna, Sweden); TPS - IRMA (IDL Biotech AB, Bromma, Sweden); TK REA (Immunotech, Prague, Czech Republic) (Table II).

Statistical evaluation. The associations of serum tumor markers and patient's prognosis were assessed for the entire cohort and then for each cancer type and also with regard to treatment (radical surgery $v s$. explorative laparotomy). Overall survival (OS) and disease-free intervals (DFI) were computed by Kaplan-Meier methods and statistically evaluated using LogRank test. DFI was computed only in the subgroup of patients that were treated by radical surgery. The tumor markers cut-off values for statistical analyses were chosen as the median of serum level of each tumor marker with respect to a limited number of subjects in the defined subgroups. 
Table III. Statistically significant results $(p<0.05)$ observed for each tumor marker in relation to disease subgroups and surgical treatment.

\begin{tabular}{|c|c|c|}
\hline Tumor marker & Disease and treatment subgroups & Significant results $(p<0.05)$ \\
\hline TK & $\begin{array}{l}\text { Gallbladder carcinoma } \\
\text { Radical surgery }\end{array}$ & $\begin{array}{c}\text { Poor prognostic for OS median OS } 0.56 v s .0 .75 \text { years } \\
\qquad \begin{array}{c}\text { HR }=4.6,95 \% \mathrm{CI}=1.0-4.7 \\
\text { cut-off }=6.3 \mathrm{IU} / 1\end{array}\end{array}$ \\
\hline TK & $\begin{array}{l}\text { Gallbladder carcinoma } \\
\text { Radical surgery }\end{array}$ & $\begin{array}{c}\text { Poor prognostic for DFI median DFI } 0.32 v s .0 .5 \text { years } \\
\text { HR }=3.5,95 \% \mathrm{CI}=0.6-21.3 \\
\text { cut-off }=6.3 \mathrm{IU} / 1\end{array}$ \\
\hline TPS & $\begin{array}{c}\text { Gallbladder carcinoma } \\
\text { All patients (radical surgery and explorative laparotomy) }\end{array}$ & $\begin{array}{c}\text { Poor prognostic for OS median OS } 0.39 v s .0 .71 \text { years } \\
\text { HR }=12.7,95 \% \text { CI } 1.4-117.7 \\
\text { cut-off }=70 \mathrm{IU} / 1\end{array}$ \\
\hline TPS & $\begin{array}{l}\text { Gallbladder carcinoma } \\
\text { Explorative laparotomy }\end{array}$ & $\begin{array}{c}\text { Poor prognostic for OS median OS } 0.37 v s .0 .69 \text { years } \\
\qquad \begin{array}{c}\text { HR }=2.1,95 \% \mathrm{CI}=0-2.9 \\
\text { cut-off }=160 \mathrm{IU} / 1\end{array}\end{array}$ \\
\hline CEA & $\begin{array}{l}\text { All cumulated diagnosis } \\
\text { Explorative laparotomy }\end{array}$ & $\begin{array}{c}\text { Poor prognostic for OS median OS } 0.38 v s .0 .77 \text { years } \\
\text { HR }=9.8,95 \% \text { CI }=1.05-92.7 \\
\text { cut-off }=2.8 \mathrm{ng} / \mathrm{ml}\end{array}$ \\
\hline
\end{tabular}

TK, Thymidine kinase; TPS, tissue polypeptide specific antigen; CEA, carcinoembryonic antigen; OS, overall survival; DFI, disease-free interval; HR, hazard ratio.

\section{Results}

We examined the potential value of tumor markers (AFP, CEA, CA 19-9 and CA 72-4), proliferative tumor marker TK and cytokeratins (CYFRA 21-1, TPA, and TPS) in the assessment of prognosis of patients with gallbladder, extrahepatic bile duct, and cholangiocellular carcinomas. The sub-analyses in each diagnosis were also done for patients treated either with radical surgery or only surgical exploration (explorative laparotomy). Only statistically significant results $(p<0.05)$ are presented (Table III). Tumor marker TK was proven as a poor prognostic factor for OS in gallbladder carcinoma patients treated with radical resection. Patients with serum TK level equal to or above cut-off value (6.3 IU/L) experienced significantly shorter OS (0.56 vs. 0.75 years, $\mathrm{HR}=4.6, p<0.05$ ) (Figure 1 ).

TPS was demonstrated to be a poor prognostic factor for OS in all patients with gallbladder carcinoma (explorative laparotomy and radical surgical treatment together). Patients with TPS serum level equal to or above cut-off value (70 IU/L) had significantly reduced OS (0.39 vs. 0.71 years, $\mathrm{HR}=12.7, p<0.05)$ (Figure 2). When divided into subgroups (radical surgery and explorative laparotomy), TPS remained the significant poor prognostic factor for OS in patients after explorative laparotomy for gallbladder carcinoma. The risk for shorter OS was higher in patients with TPS serum level equal to or above $160 \mathrm{IU} / \mathrm{L}$ cut-off value $(0.37$ vs. 0.69 years, $\mathrm{HR}=2.1, p<0.05$ ) (Figure 3). The preoperative serum level of CEA was demonstrated to be an interesting poor prognostic factor for OS in all patients (gallbladder, extrahepatic bile duct, and cholangiocellular carcinomas altogether) experiencing explorative laparotomy. Patients with serum CEA level equal to or above cut-off value $(2.8 \mathrm{ng} / \mathrm{ml})$ experienced significantly shorter OS (0.38 vs. 0.77 years, HR=9.8, $p<0.05)$ (Figure 4). The analyses of preoperative serum levels of other tumor markers in relation to the patient prognosis and OS did not reach statistical significance.

The preoperative serum level of TK was proven to be a poor prognostic factor for DFI in patients after radical surgical treatment of gallbladder carcinoma $(p<0.05)$ (Figure 5). Patients with TK serum level equal to or above cut-off value $(6.3 \mathrm{IU} / \mathrm{l})$ had significantly shorter DFI (0.32 vs. 0.5 years, $\mathrm{HR}=3.5, p<0.05)$. The analyses of preoperative serum levels of other tumor markers in relation to the patient DFI did not reach statistical significance.

\section{Discussion}

This pilot study of tumor markers (AFP, CA 1-9, CA 72-4, CEA, CYFRA, TK, TPA, and TPS) in biliary tract malignancies revealed TK as a significant poor prognostic factor for OS and also DFI in patients after radical resection for gallbladder carcinoma. The preoperative serum level of TPS was proven to be a poor prognostic factor for OS in all patients with gallbladder carcinoma (the cumulative subgroup of radical surgery and explorative laparotomy). TPS was also demonstrated as a poor prognostic factor for OS in the subgroup of patients with only explorative laparotomy for gallbladder carcinoma. Preoperative serum level of CEA was observed as a poor prognostic factor for OS in patients experiencing explorative laparotomy for all cumulated biliary tract malignancies. 


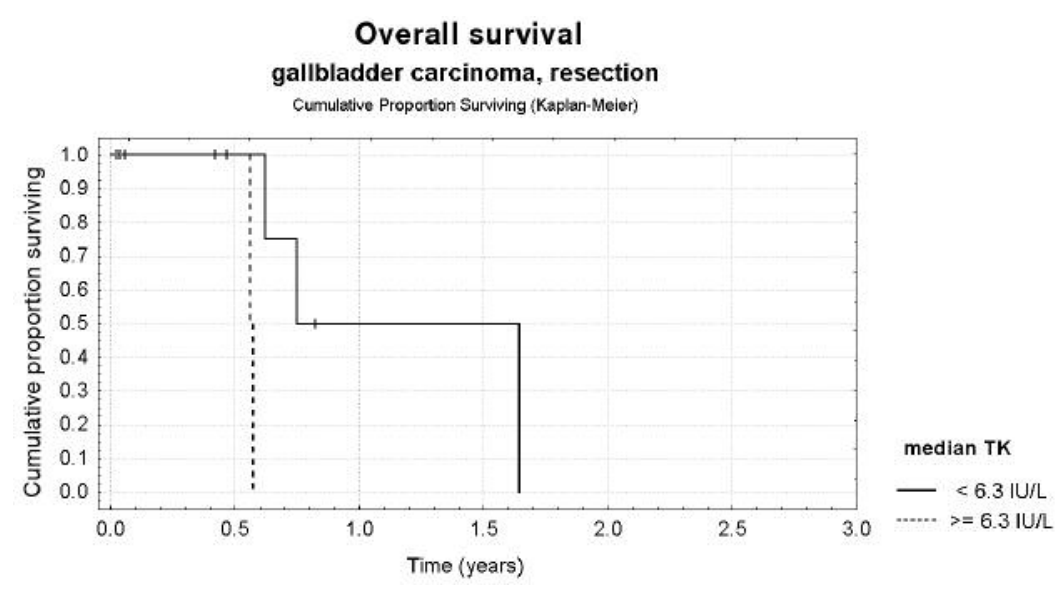

Figure 1. Preoperative serum level of TK (IU/l) as a poor prognostic factor for OS in patients after radical resection for gallbladder carcinoma (0.56 vs. 0.75 years, $H R=4.6, p<0.05$ ).

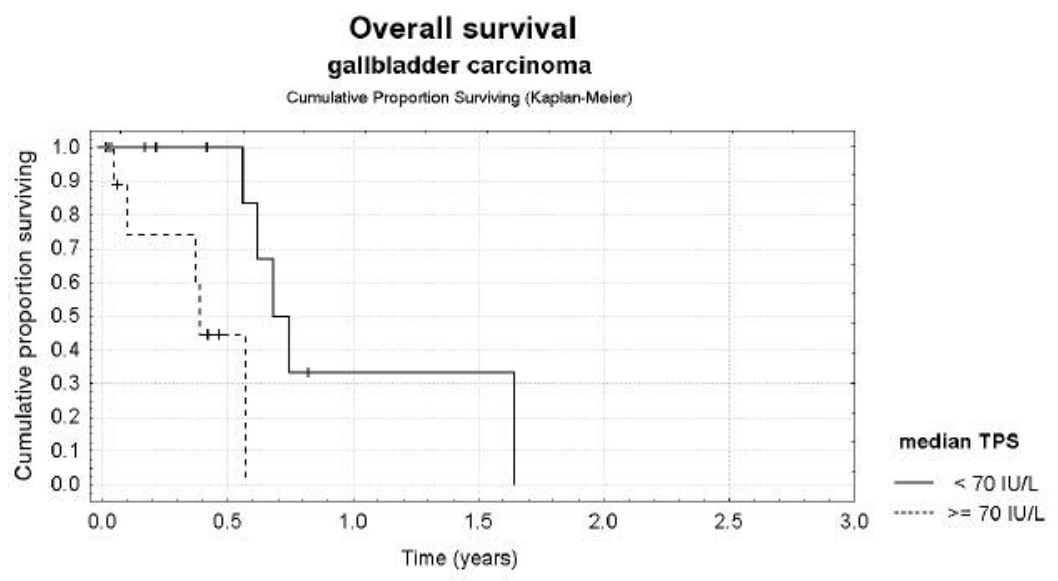

Figure 2. Preoperative serum level of TPS (IU/L) as a prognostic factor for OS in all patients with gallbladder carcinoma (0.39 vs. 0.71 years, $H R=12.7, p<0.05)$.

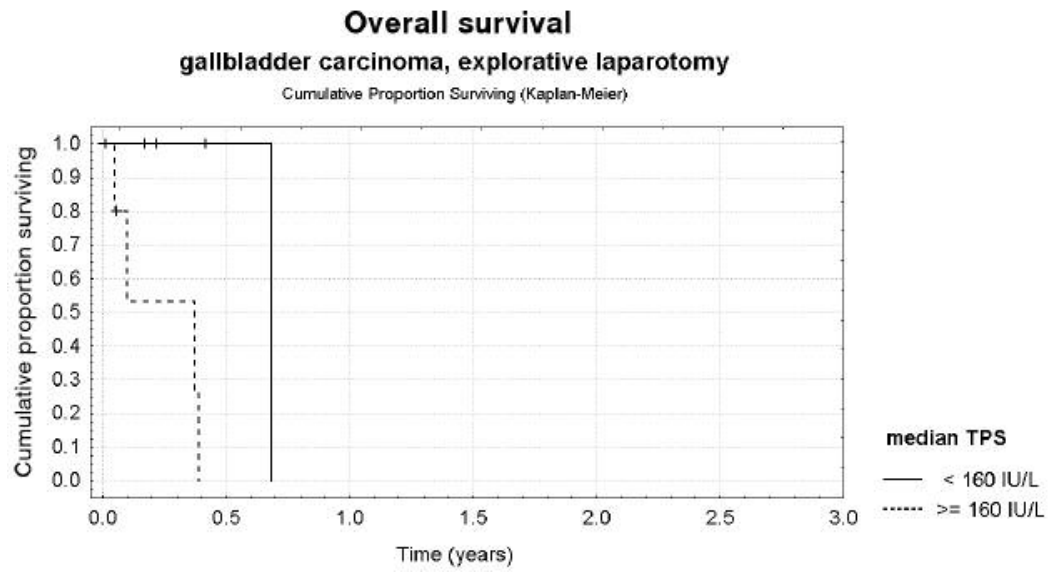

Figure 3. Preoperative serum level of TPS (IU/l) as a poor prognostic factor for OS in patients after explorative laparotomy for gallbladder carcinoma (0.37 vs. 0.69 years, $H R=2.1, p<0.05)$. 


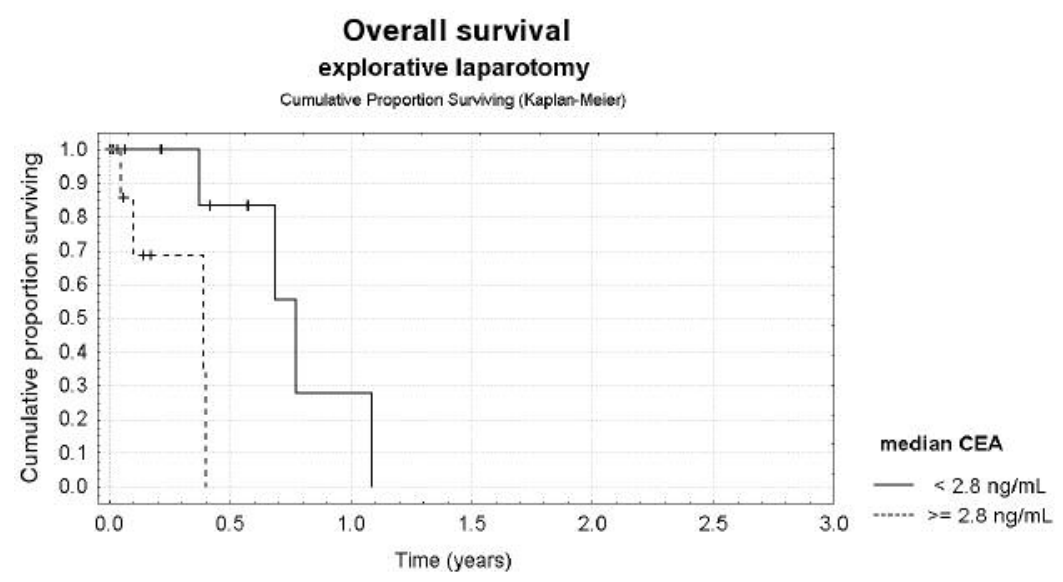

Figure 4. Preoperative serum level of CEA $(\mathrm{ng} / \mathrm{ml})$ as a poor prognostic factor for OS in patients after explorative laparotomy for all cumulated studied diagnosis (0.38 vs. 0.77 years, $H R=9.8, p<0.05$ ).

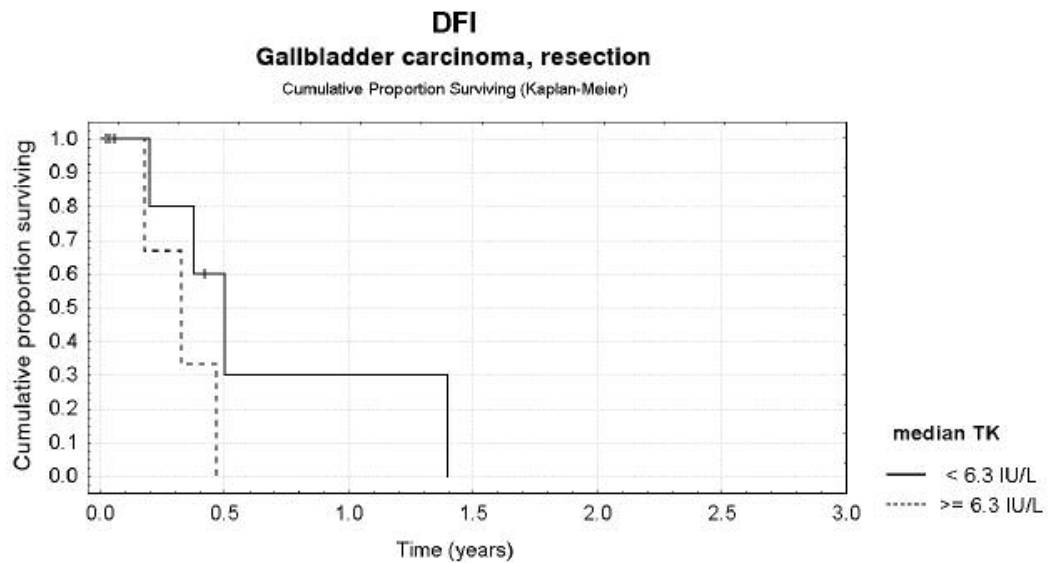

Figure 5. Preoperative serum level of TK $(I U / L)$ as a poor prognostic factor for DFI in patients after radical surgical treatment of gallbladder carcinoma (0.32 vs. 0.5 years, $H R=3.5, p<0.05)$.

Tumor markers (CA 19-9 and CA 125) and their role in patients with cholangitis and bile duct carcinoma were originally examined by Kerr et al. (11). Patients with bile duct carcinoma $(n=18)$ compared to those with acute cholangitis $(n=25)$ had higher positivity rate of serum CA19$9(61.1 \%$ vs. $28.0 \%)$ as well as CA125 (27.78\% vs. $4 \%)$. The study demonstrated a diagnostic accuracy of $67.4 \%$ in bile duct carcinoma for both the CA19-9 and CA125. Brockman et al. (12) examined the concentrations of tumor markers CA 19-9, CEA, CA 72-4, CA125 and AFP in bile from resected gallbladders of patients suffering from pancreato-biliary system carcinomas by comparison with patients suffering from acute cholecystitis, cholecystolithiasis, and benign pancreato-biliary tumors. Sensitivity reached $100 \%$ for patients with bile duct carcinoma (CA 19-9) and papillary carcinoma (CEA) at a specificity of $100 \%$. The associations of CEA, CA19-9, and CA72-4 with a prognosis of patients with gallbladder carcinoma was observed, but the study comprised only 7 gall bladder tumors. Another study assessed the serums levels of tumor markers AFP, CA19-9, CA125 and CEA in patients with cholangiocarcinoma $(n=30)$ and patients with hepatocellular carcinoma $(n=30)(13)$. Serum levels of CA19-9, CA125 and CEA were significantly higher and AFP level was lower in cholangiocarcinoma compared to hepatocellular carcinoma patients. The area under the receiver operating characteristic (ROC) curve was 0.94 (95\%CI=0.880.99 ) for the tumor markers combination in the diagnosis of cholangiocarcinoma. The Recent meta-analysis of 31 studies comprising 1,264 cholangiocarcinoma patients and 2,039 controls revealed the diagnostic value of tumor marker CA199 for this disease with the ROC number of 0.83 (14). The serum level of CA19-9 has also associated with prognosis of 84 distal cholangiocarcinoma patients experienced radical surgery with curative intent (15). Patients with lower CA19- 
9 level $(<150 \mathrm{U} / \mathrm{l})$ had significantly higher OS rates $(\mathrm{p}<0.05)$. Another recent study with patients suffering from advanced and inoperable bile duct cancers (212 patients in the investigational cohort and 677 patients in tree independent validation cohorts) showed that the decline of CA 19-9 serum level during chemotherapy was predictive for longer OS $(\mathrm{HR}=0.60,95 \% \mathrm{CI}=0.44-0.80, p<0.0005)(16)$. Similarly, the decline of CA19-9 and CEA $\geq 30 \%$ after the first cycle of S-1 and cisplatin chemotherapy in 104 patients with metastatic or relapsed biliary tract cancers served as a positive prognostic factor of time to progression ( $\mathrm{HR}=0.44$, $p=0.003)$ as well as $\mathrm{OS}(\mathrm{HR}=0.37, p<0.001)$ in the multivariate analysis (17). In another recent study with biliary tract cancer patients undergoing resection with curative intent, the worse 3-year OS was observed among patients with an abnormally high $(\geq 37 \mathrm{U} / \mathrm{ml})$ preoperative as well as postoperative CA19-9 levels by comparison with those with either the high preoperative and normal postoperative CA199 levels, or normal preoperative CA19-9 level (3-year OS rate 31,73 , and $70.4 \%$ respectively, $p<0.001$ ) (18). The postoperative CA19-9 level $(<37$ vs. $\geq 37 \mathrm{U} / \mathrm{ml})$ was an independent prognostic factor for a long-term survival $(\mathrm{HR}=7.296, p=0.002)$ in the study with 97 patients with the extrahepatic bile duct cancers as well (19).

There is still a relative lack of studies with common tumor markers in biliary tract malignancies in relation to patient prognosis, which is mainly caused by the rare incidence of these tumors as well as the long time required for an appropriate cohort collection.

Moreover, recognition of the clinical scenario for which the tumor markers are assessed is crucial for the validity of results. The interpretation of tumor markers depends on the art of processing, which should be chosen with regard to "the clinical question asked" $(20,21)$. The recommendations for clinical utility of tumor markers stated by European Group on Tumor Markers (EGTM) or American Society of Clinical Oncology (ASCO) deal predominantly with the most common malignancies despite the fact that tumor markers could play a substantial role in rarer types of cancers as well (22-24). As was demonstrated in this study, the preoperative TPS serum level is an important prognostic factor for OS in patients with gallbladder carcinoma. The assessment of TPS serum level before the operation could help surgeons with the decision about the most suitable extent of surgery (radical resection or only explorative laparotomy) for an individual patient with the high risk of shorter survival irrespective of the surgical intervention applied.

The main limitation of this study remains the relatively small number of enrolled patients, particularly in the subgroup analyses regarding distinct cancer types and different surgical treatment strategies. In spite of this limitation, presented results are promising and illustrate the importance of common tumor markers in diagnostic and treatment decision strategies in patients with biliary tract malignancies. Further research in this field with expanded cohorts of patients is highly desirable.

\section{Conclusion}

The results of this pilot study of serum tumor markers in biliary tract malignancies revealed the preoperative level of TPS as a poor prognostic factor for OS in patients with gallbladder carcinoma. Preoperative serum level of CEA was proved as a poor prognostic factor for OS in patients after explorative laparotomy for all cumulated biliary tract malignancies. Preoperative serum level of TK was demonstrated as a poor prognostic factor for OS and also DFI in patients after radical resection for gallbladder carcinoma. Presented results demonstrate the importance of common tumor markers in diagnostic and treatment decision strategies in patients with biliary tract malignancies.

\section{Acknowledgements}

This work was supported by the National Sustainability Program I (NPU I) Nr. LO1503 provided by the Ministry of Education Youth and Sports of the Czech Republic.

\section{References}

1 Mekeel KL and Hemming AW: Surgical management of gallbladder carcinoma: a review. J Gastrointest Surg 11: 11881193, 2007.

2 Leonard GD and O'Reilly EM: Biliary tract cancers: current concepts and controversies. Expert Opin Pharmacother 6: 211223, 2005.

3 Park JO, Oh D-Y, Hsu C, Chen J-S, Chen L-T, Orlando M, Kim JS and Lim HY: Gemcitabine plus cisplatin for advanced biliary tract cancer: a systematic review. Cancer Res Treat 47: 343-361, 2015.

4 Macias RIR: Cholangiocarcinoma: biology, clinical management, and pharmacological perspectives. ISRN Hepatol 2014: 828074, 2014.

5 Bosch FX, Ribes J, Díaz M and Cléries R: Primary liver cancer: worldwide incidence and trends. Gastroenterology 127: S5-S16, 2004.

6 Balachandran P, Agarwal S, Krishnani N, Pandey CM, Kumar A, Sikora SS, Saxena R and Kapoor VK: Predictors of long-term survival in patients with gallbladder cancer. J Gastrointest Surg 10: 848-854, 2006.

7 Yagi H, Shimazu M, Kawachi S, Tanabe M, Aiura K, Wakabayashi G, Ueda M, Nakamura Y and Kitajima M: Retrospective analysis of outcome in 63 gallbladder carcinoma patients after radical resection. J Hepatobiliary Pancreat Surg 13: 530-536, 2006.

8 Roskams T and Desmet V: Embryology of extra- and intrahepatic bile ducts, the ductal plate. Anat Rec Hoboken 291: 628-635, 2008.

9 Suchy FJ: Clinical problems with developmental anomalies of the biliary tract. Semin Gastrointest Dis 14: 156-164, 2003.

10 Geller SA, Dhall D and Alsabeh R: Application of immunohistochemistry to liver and gastrointestinal neoplasms: liver, stomach, colon, and pancreas. Arch Pathol Lab Med 132: 490-499, 2008. 
11 Ker CG, Chen JS, Lee KT, Sheen PC and Wu CC: Assessment of serum and bile levels of CA19-9 and CA125 in cholangitis and bile duct carcinoma. J Gastroenterol Hepatol 6: 505-508, 1991.

12 Brockmann J, Emparan C, Hernandez CA, Sulkowski U, Dietl KH, Menzel J, Wolters H, Glodny B and Senninger N: Gallbladder bile tumor marker quantification for detection of pancreato-biliary malignancies. Anticancer Res 20: 4941-4947, 2000.

13 Li Y, Li D-J, Chen J, Liu W, Li J-W, Jiang P, Zhao X, Guo F, Li X-W and Wang S-G: Application of Joint Detection of AFP, CA19-9, CA125 and CEA in Identification and Diagnosis of Cholangiocarcinoma. Asian Pac J Cancer Prev 16: 3451-3455, 2015

14 Liang B, Zhong L, He Q, Wang S, Pan Z, Wang T and Zhao Y: Diagnostic accuracy of serum CA19-9 in patients with cholangiocarcinoma: a systematic review and meta-analysis Med Sci Monit 21: 3555-3563, 2015.

15 Tan X, Xiao K, Liu W, Chang S, Zhang $\mathrm{T}$ and Tang $\mathrm{H}$ : Prognostic factors of distal cholangiocarcinoma after curative surgery: a series of 84 cases. Hepatogastroenterology 60: 18921895,2013

16 Grunnet M, Christensen IJ, Lassen U, Jensen LH, Lydolph M, Knox JJ, McNamara MG, Jitlal M, Wasan H, Bridgewater J, Valle JW and Mau-Sorensen M: Decline in CA19-9 during chemotherapy predicts survival in four independent cohorts of patients with inoperable bile duct cancer. Eur J Cancer 51: 13811388, 2015.

17 Lee D-W, Im S-A, Kim YJ, Yang Y, Rhee J, Na II, Lee K-H, Kim T-Y, Han S-W, Choi IS, Oh D-Y, Kim JH, Kim T-Y and Bang Y-J: CA19-9 or CEA Decline after the First Cycle of Treatment Predicts Survival in Advanced Biliary Tract Cancer Patients Treated with S-1 and Cisplatin Chemotherapy. Cancer Res Treat, 2017. doi: 10.4143/crt.2016.326
18 Yamashita S, Passot G, Aloia TA, Chun YS, Javle M, Lee JE, Vauthey J-N and Conrad C: Prognostic value of carbohydrate antigen 19-9 in patients undergoing resection of biliary tract cancer. Br J Surg 104: 267-277, 2017.

19 Lee HS, Lee SH, Roh YH, Chung MJ, Park JY, Park SW, Song SY, Chung JB and Bang S: Efficacy of Adjuvant Chemotherapy and Prognostic Factors for Patients with Extrahepatic Bile Duct Cancer. Chemotherapy 61: 152-158, 2016.

20 Bidart JM, Thuillier F, Augereau C, Chalas J, Daver A, Jacob N, Labrousse $\mathrm{F}$ and Voitot $\mathrm{H}$ : Kinetics of serum tumor marker concentrations and usefulness in clinical monitoring. Clin Chem 45: 1695-1707, 1999.

21 Duffy MJ: Role of tumor markers in patients with solid cancers: A critical review. Eur J Intern Med 18: 175-184, 2007.

22 Duffy MJ, van Dalen A, Haglund C, Hansson L, Holinski-Feder E, Klapdor R, Lamerz R, Peltomaki P, Sturgeon C and Topolcan $\mathrm{O}$ : Tumour markers in colorectal cancer: European Group on Tumour Markers (EGTM) guidelines for clinical use. Eur J Cancer 43: 1348-1360, 2007.

23 Locker GY, Hamilton S, Harris J, Jessup JM, Kemeny N, Macdonald JS, Somerfield MR, Hayes DF, Bast RC and ASCO: ASCO 2006 update of recommendations for the use of tumor markers in gastrointestinal cancer. J Clin Oncol 24: 5313-5327, 2006.

24 Duffy MJ, Sturgeon CM, Sölétormos G, Barak V, Molina R, Hayes DF, Diamandis EP and Bossuyt PMM: Validation of new cancer biomarkers: a position statement from the European group on tumor markers. Clin Chem 61: 809-820, 2015.

Received February 7, 2017

Revised March 7, 2017

Accepted March 9, 2017 